\title{
Effectiveness of some Insecticides for Eggplant Sucking-Piercing Insects Management with Reference to Their Side Effects on Natural Enemies
}

\author{
Hamdy K. Abou-Taleb ${ }^{1}$ and Ahmed A. Barrania ${ }^{2}$
}

\begin{abstract}
Two field experiments were conducted during 2012 and 2013 seasons at Nubarya district, to evaluate one synthetic insecticide, four insecticide alternatives and their binary mixtures against some eggplant sucking insects. The side effect of these insecticide treatments against two predatory insects was also investigated. Results showed that, Admire, Admire-Achook mixture, and AdmireApplaud mixture achieved the highest reduction percentages of whitefly, where mean reduction percentages were $93.4,91.7$ and $93.3 \%$ at 2012 and 94.3, 95.1 and $94.1 \%$ at 2013 , respectively. In the case of aphids, in 2012 and 2013 seasons, the highest reduction percentages were achieved by Admire (98.6 and 98.2\%), Kemesol (95.2 and 94.9\%), Admire-Kemesol mixture (98.9 and 99.4\%), Admire-Achook mixture (98.6 and 99.1\%) and Admire-Applaud mixture (96.9 and 95.8\%). Concerning jassid, it was recorded that Admire and Admire-Achook mixture treatments achieved the highest reduction followed by Admire-Kemesol mixture. In both seasons, Admire has the highest negative effect on the lady beetle, Coccinella sp. and aphid lion, Chrysopa spp. Admire reduced lady beetle by 59.8 and $57.7 \%$ in 2012 and 2013, respectively and reduced aphid lion by 25.5 and $22.6 \%$ in 2012 and 2013, respectively. On the other hand, Breef oil has the least side effects on the two predators in both seasons. Finally we can conclude that, Admire-Achook mixture can be used for sucking-piercing insects management on eggplant, where this mixture achieved the highest reduction percentages against the insect pests and relative low ones against natural enemies.
\end{abstract}

\section{INTRODUCTION}

Eggplant (Solanum melanogena L.) is an important vegetable crop grown in various tropical and temperate parts of the world (Kashyap et al., 2003). It is a good source of vitamins and minerals (particularly iron) making its total nutritional value comparable with tomato (Kalloo, 1993). Eggplant has been used in traditional medicines (Khan, 1979). For example, tissue extracts have been used for treatment of asthma, bronchitis, cholera and dysuria; fruits and leaves are beneficial in lowering blood cholesterol. Whitefly, Bemisia tabaci (Genn.), aphid, Aphis gossypii Glover, and jassid, Emboasca lybica deBerg, are between the most important insect pests of eggplant (Yadav and Kumawat, 2013). These insect pests are attacking a wide spectrum of economic plants, causing greet losses in their yield. The problems of these insects are not only due to its direct damage to the infested plants but also to its capability to transmit viruses.

The continuous and unwise uses of insecticides by farmers usually lead to adverse effects on naturally occurring biological control agents and the biotic environment as well. Also, eggplant can be infected by insect pests during the harvesting period. Therefore, the search for more safe insecticide alternatives is becoming so urgent. So, the aim of this work was to evaluate some insecticide alternatives in comparison with the synthetic insecticide Admire against the three insect pests as well as two natural enemies. In addition, the mixtures of each of these alternatives with Admire (each at half field rates) were also evaluated.

\section{MATERIALS AND METHODS}

Tested insecticides and control agents: The evaluated insecticides and control agents, common names and rates were introduced in the following Table (1):

Field experiments: Two experiments were conducted during 2012 and 2013 seasons, at Nubaryia district. Eggplant seedlings were transplanted at March 20, 2012 and March 18, 2013. Treatments were arranged in a randomized complete block design (RCBD). Each treatment was replicated four times $\left(84 \mathrm{~m}^{2}\right.$ per each). The insecticides were sprayed by Knapsack sprayer equipment (CP3) at the rate of 200 liter per feddan on May 1, 2012 and May 2, 2013, respectively. Control was sprayed only by water.

The efficiency of the tested products was estimated by counting of the target alive insects on the lower surface of ten eggplant leaves per each plot. Pretreatment counts were done just before application while post-treatment counts were made on days $1,3,5$, 7, 9 and 12 days after treatment. Counts were done in the early morning when flight activity is minimal according to Bulter et al. (1988). Reduction percentages were calculated according to Henderson and Tilton equation (1955). The treatments were compared with each other using one way ANOVA with $\mathrm{LSD}_{0.05}$ (CoStat Statistical Software, 1990).

\footnotetext{
${ }^{1}$ Plant Protection Research Institute, ARC, Sabahia,

Alexandria, Egypt.

${ }^{2}$ Plant Protection Research Institute, Etay El-baroud Agric.

Res. Station. Agric. Res. Center, Egypt.

Received May 11, 2014, Accepted June28, 2014
} 
Table 1. insecticides and control agents treatments, common names and field rates

\begin{tabular}{lcc}
\hline Trade name & Common name & Rate / 100 liter water \\
\hline Admire 20\% SC & Imidacloprid & $125 \mathrm{ml}$ \\
\hline Kemesol 95\% EC & Mineral oil & $1000 \mathrm{ml}$ \\
\hline Achook 0.15\% EC & Azadirachtin & $250 \mathrm{ml}$ \\
\hline Applaud 25\% SC & buprofezin & $200 \mathrm{ml}$ \\
\hline Breef & Orange oil & $500 \mathrm{ml}$ \\
\hline Admire + Kemesol & & $65 \mathrm{ml}+500 \mathrm{ml}$ \\
\hline Admire + Achook & & $65 \mathrm{ml}+125 \mathrm{ml}$ \\
\hline Admire + Applaud & & $65 \mathrm{ml}+100 \mathrm{ml}$ \\
\hline Admire + Breef & & $65 \mathrm{ml}+250 \mathrm{ml}$ \\
\hline
\end{tabular}

\section{RESULTS AND DISCUSSION}

Field efficiency of some insecticide treatments against whitefly on the eggplant: Gradual reduction percentages of whitefly numbers as a result of insecticide treatments were recorded in both seasons 2012 and 2013 (Tables 2.A and 2.B). The highest reduction percentages of whitefly were recorded by Admire, Admire-Achook mixture, and Admire-Applaud mixture, where mean reduction percentages were 93.4 , 91.7 and $93.3 \%$ at 2012 and $94.3,95.1$ and $94.1 \%$ at 2013, respectively. The least reduction percentages were recorded by Breef and Admire-Breef mixture at the two seasons (2012 and 2013). The same gradual increasing in reduction percentages in whitefly numbers was recorded with the nicotinic acetylcholine receptor agonist Confidor (imidacloprid), which was potent on days 7 and 14 after application as recorded by Motohiro and Casida (2003); Horowitz et al. (1998). Our results were comparable with El Bessomy (2003), where he reported that Admire (imidacloprid) gave good reduction percentages against the whitefly after $84 \mathrm{hrs}$ of application.

Field efficiency of some insecticide treatments against aphids on the eggplant: Field evaluation of some insecticides treatments against aphids on the eggplant at 2012 and 2013 seasons was carried out (Tables 3. A \& B). In both seasons 2012 and 2013, the highest reduction percentages were achieved by Admire (98.6 and 98.2\%), Kemesol (95.2 and 94.9\%), AdmireKemesol mixture (98.9 and 99.4\%), Admire-Achook mixture (98.6 and 99.1\%) and Admire-Applaud mixture (96.9 and 95.8\%). These results are in agreement with Abdel Rahman and Abou-Taleb, (2008), where they reported that, Confidor (imidacloprid) was the most effective in controlling aphids on cotton plants. Also, Shehata et al. (2009) mentioned that Confidor achieved the highest aphid control on the cucumber plants.

Field efficiency of some insecticide treatments against jassid on the eggplant: Reduction percentages of jassid as a result of some insecticide treatments on eggplants at 2012 and 2013 seasons are presented in Tables (4. A \& B). In 2012, Admire and AdmireAchook mixture treatments achieved the highest effectiveness followed by Admire-Kemesol mixture with mean reduction percentages of $89.7,87.1$ and $81.5 \%$, respectively (Table $4 . \mathrm{A}$ ). The same trend was observed in 2013 (Table 4.B). Admire and AdmireAchook mixture treatments caused jassid reduction percentages of 89.7 and $86.7 \%$, respectively. These results were comparable with results of many authors. Aslam et al. (2004) reported that, between seven insecticides tested against cotton sucking insects in Pakistan, Confidor ${ }^{\mathbb{R}}$ was the most effective in controlling jassid and thrips. Also, Wahla et al. (1997) and Hameed et al. (1997) investigated that Confidor ${ }^{\circledR}$ effectively controlled thrips and jassid in cotton.

Side effects of some insecticide treatments on the lady beetle and Aphid lion: Probably the most common pest management activity that negatively impacts beneficial organisms in agroecosystems is pesticide application. As a result, modifications of pesticide use practices are the most commonly implemented form of conservation biological control, and have long been considered an important component of integrated pest management programs (Ruberson et al., 1998). Lady beetles are known to be voracious predators of plant pests such as aphids (Gordon 1985). It is often assumed that aphidophagous lady beetles are highly polyphagous, consuming most aphid species that they encounter (Pedigo and Rice 2006). Also, Chrysopidae lacewings family has been amongst useful insects of agricultural ecosystems which are very effective and practical in biological control programs against agricultural pests (Canard et al. 1984). This family included more than 90 types and 1800 wellknown species which their predating behavior always attracted the entomologists' intention in biological control programs (Brook and Barnard, 1990).

In this study, the side effects of insecticide treatments on the two predators, lady beetle and aphid 
lion was investigated and presented in Tables (5. A \& B and 6. A \& B). In both seasons, Admire has the highest negative effect on the lady beetle and aphid lion. Admire reduced lady beetle by 59.8 and $57.7 \%$ compared to control in 2012 and 2013, respectively (Tables 5. A \& B) and reduced aphid lion by 25.5 and $22.6 \%$ compared to control in 2012 and 2013, respectively (Tables 6. A \& B). On the other hand, Breef oil has the least side effects on the two predators in both seasons. Mixtures of Admire with Achoock or Applaud or Breef (each at a half field rate) have a moderate side effects against the two predators in both seasons.

Table 2. A. Efficacy of certain treatments against whitfly on the eggplant (season 2012)

\begin{tabular}{lccccccc}
\hline \multirow{2}{*}{ Treatments } & \multicolumn{9}{c}{ \% Reduction } & \multicolumn{2}{c}{ Mean } \\
\cline { 2 - 7 } & 1-day & 3-days & 5-days & 7-days & 9-days & 12-days & \\
\hline Admire & 81.2 & 93.1 & 97.3 & 98.2 & 95.4 & 95.2 & $93.4 \mathrm{a}$ \\
\hline Kemesol & 56.5 & 75.4 & 75.2 & 80.0 & 78.3 & 78.1 & $73.9 \mathrm{c}$ \\
\hline Achook & 50.7 & 70.1 & 82.6 & 83.8 & 79.9 & 80.5 & $74.6 \mathrm{bc}$ \\
\hline Applaud & 40.5 & 62.3 & 83.5 & 88.6 & 91.3 & 88.4 & $75.8 \mathrm{bc}$ \\
\hline Breef & 34.4 & 47.2 & 58.7 & 68.3 & 71.4 & 75.6 & $61.7 \mathrm{~d}$ \\
\hline Admire + Kemesol & 75.1 & 77.3 & 80.2 & 80.9 & 83.4 & 79.0 & $79.3 \mathrm{~b}$ \\
\hline Admire + Achook & 80.9 & 88.3 & 95.9 & 95.8 & 95.5 & 93.7 & $91.7 \mathrm{a}$ \\
\hline Admire + Applaud & 83.7 & 89.1 & 97.2 & 96.6 & 97.0 & 96.3 & $93.3 \mathrm{a}$ \\
\hline Admire + Breef & 50.5 & 60.4 & 67.3 & 72.9 & 72.6 & 71.8 & $65.9 \mathrm{~d}$ \\
\hline
\end{tabular}

Means followed by the same letters are not significantly different according to the $\mathrm{LSD}_{0.05}$.

Table 2.B. Efficacy of certain treatments against whitfly on the eggplant (season 2013)

\begin{tabular}{lccccccc}
\hline Treatments & \multicolumn{9}{c}{ \% Reduction } & \multirow{2}{*}{ Mean } \\
\cline { 2 - 6 } & 1-day & 3-days & 5-days & 7-days & 9-days & 12-days & \\
\hline Admire & 85.5 & 95.7 & 97.9 & 98.3 & 95.2 & 93.2 & $94.3 \mathrm{a}$ \\
Kemesol & 60.6 & 79.1 & 80.3 & 80.5 & 77.4 & 77.3 & $75.9 \mathrm{c}$ \\
Achook & 52.2 & 76.1 & 84.7 & 85.5 & 81.6 & 82.7 & $77.1 \mathrm{c}$ \\
Applaud & 49.3 & 71.5 & 89.2 & 88.9 & 90.3 & 92.6 & $80.3 \mathrm{bc}$ \\
Breef & 40.5 & $55 . .6$ & 63.1 & 69.8 & 70.9 & 72.2 & $63.3 \mathrm{~d}$ \\
Admire + Kemesol & 77.8 & 82.1 & 84.5 & 85.6 & 86.7 & 83.8 & $83.4 \mathrm{~b}$ \\
Admire + Achook & 84.6 & 97.3 & 97.1 & 97.7 & 96.9 & 97.0 & $95.1 \mathrm{a}$ \\
Admire + Applaud & 84.2 & 93.6 & 97.0 & 98.4 & 96.3 & 95.2 & $94.1 \mathrm{a}$ \\
Admire + Breef & 50.5 & 58.6 & 65.5 & 70.1 & 72.3 & 70.4 & $64.6 \mathrm{~d}$ \\
\hline
\end{tabular}

Means followed by the same letters are not significantly different according to the $\mathrm{LSD}_{0.05}$.

Table 3.A. Efficacy of certain treatments against aphids on the eggplant (season 2012)

\begin{tabular}{|c|c|c|c|c|c|c|c|}
\hline \multirow[t]{2}{*}{ Treatments } & \multicolumn{6}{|c|}{ \% Reduction } & \multirow[t]{2}{*}{ Mean } \\
\hline & 1-day & 3-days & 5-days & 7-days & 9-days & 12-days & \\
\hline Admire & 93.6 & 98.2 & 100.0 & 100.0 & 100.0 & 100.0 & $98.6 \mathrm{a}$ \\
\hline Kemesol & 80.6 & 95.8 & 96.3 & 98.5 & 100.0 & 100.0 & $95.2 \mathrm{a}$ \\
\hline Achook & 78.0 & 84.2 & 88.7 & 93.5 & 92.8 & 94.4 & $88.6 \mathrm{~b}$ \\
\hline Applaud & 76.3 & 80.1 & 89.7 & 90.8 & 90.0 & 92.9 & $86.6 \mathrm{~b}$ \\
\hline Breef & 44.6 & 49.5 & 52.5 & 60.1 & 66.9 & 68.3 & $57.0 \mathrm{c}$ \\
\hline Admire + Kemesol & 95.4 & 98.1 & 100.0 & 100.0 & 100.0 & 100.0 & $98.9 \mathrm{a}$ \\
\hline Admire + Achook & 94.2 & 97.3 & 100.0 & 100.0 & 100.0 & 100.0 & $98.6 \mathrm{a}$ \\
\hline Admire + Applaud & 90.5 & 93.2 & 97.5 & 100.0 & 100.0 & 100.0 & $96.9 \mathrm{a}$ \\
\hline Admire + Breef & 80.6 & 87.4 & 90.0 & 90.1 & 92.5 & 91.3 & $88.7 \mathrm{~b}$ \\
\hline
\end{tabular}

Means followed by the same letters are not significantly different according to the $\operatorname{LSD}_{0.05}$. 


\begin{tabular}{|c|c|c|c|c|c|c|c|}
\hline \multirow[t]{2}{*}{ Treatments } & \multicolumn{6}{|c|}{ \% Reduction } & \multirow[t]{2}{*}{ Mean } \\
\hline & 1-day & 3-days & 5-days & 7-days & 9-days & 12-days & \\
\hline Admire & 92.4 & 96.7 & 100.0 & 100.0 & 100.0 & 100.0 & $98.2 \mathrm{a}$ \\
\hline Kemesol & 82.5 & 92.8 & 96.3 & 97.9 & 100.0 & 100.0 & $94.9 \mathrm{ab}$ \\
\hline Achook & 81.4 & 87.1 & 91.7 & 94.2 & 96.9 & 95.1 & $91.1 \mathrm{bc}$ \\
\hline Applaud & 73.5 & 78.8 & 85.1 & 88.6 & 91.2 & 92.0 & $84.9 \mathrm{~d}$ \\
\hline Breef & 42.5 & 47.8 & 50.1 & 57.3 & 67.8 & 68.1 & $55.6 \mathrm{e}$ \\
\hline Admire + Kemesol & 96.5 & 100.0 & 100.0 & 100.0 & 100.0 & 100.0 & $99.4 \mathrm{a}$ \\
\hline Admire + Achook & 96.2 & 98.5 & 100.0 & 100.0 & 100.0 & 100.0 & $99.1 \mathrm{a}$ \\
\hline Admire + Applaud & 88.6 & 93.0 & 95.2 & 97.8 & 100.0 & 100.0 & $95.8 \mathrm{a}$ \\
\hline Admire + Breef & 80.3 & 86.1 & 89.9 & 91.2 & 90.6 & 90.7 & $88.1 \mathrm{~cd}$ \\
\hline
\end{tabular}

Means followed by the same letters are not significantly different according to the $\mathrm{LSD}_{0.05}$.

Table 4.A. Efficacy of certain treatments against jassid on the eggplant (season 2012)

\begin{tabular}{|c|c|c|c|c|c|c|c|}
\hline \multirow{2}{*}{ Treatments } & \multicolumn{6}{|c|}{$\%$ Reduction } & \multirow[t]{2}{*}{ Mean } \\
\hline & 1-day & 3-days & 5-days & 7-days & 9-days & 12-days & \\
\hline Admire & 85.3 & 88.2 & 90.6 & 90.3 & 92.8 & 91.1 & $89.7 \mathrm{a}$ \\
\hline Kemesol & 55.0 & 55.6 & 58.8 & 59.1 & 61.7 & 60.9 & $58.5 \mathrm{~cd}$ \\
\hline Achook & 42.6 & 45.2 & 50.8 & 50.1 & 51.4 & 48.9 & $48.2 \mathrm{e}$ \\
\hline Applaud & 37.2 & 39.6 & 38.9 & 40.1 & 40.1 & 41.6 & $39.6 \mathrm{f}$ \\
\hline Breef & 35.9 & 41.7 & 44.9 & 46.0 & 45.2 & 46.6 & $43.4 \mathrm{f}$ \\
\hline Admire + Kemesol & 72.4 & 80.6 & 83.8 & 85.1 & 83.0 & 83.9 & $81.5 \mathrm{~b}$ \\
\hline Admire + Achook & 79.3 & 86.2 & 87.9 & 89.0 & 91.4 & 88.8 & $87.1 \mathrm{a}$ \\
\hline Admire + Applaud & 52.6 & 59.1 & 64.2 & 62.1 & 64.2 & 66.7 & $61.5 \mathrm{c}$ \\
\hline Admire + Breef & 50.6 & 54.3 & 55.1 & 55.7 & 54.9 & 55.0 & $54.3 \mathrm{~d}$ \\
\hline
\end{tabular}

Means followed by the same letters are not significantly different according to the $\mathrm{LSD}_{0.05}$.

Table 4.B. Efficacy of certain treatments against jassid on the eggplant (season 2013)

\begin{tabular}{|c|c|c|c|c|c|c|c|}
\hline \multirow[t]{2}{*}{ Treatments } & \multicolumn{6}{|c|}{$\%$ Reduction } & \multirow[t]{2}{*}{ Mean } \\
\hline & 1-day & 3 -days & 5-days & 7-days & 9-days & 12-days & \\
\hline Admire & 83.8 & 86.5 & 91.0 & 92.7 & 93.4 & 90.6 & $89.7 \mathrm{a}$ \\
\hline Kemesol & 59.5 & 62.3 & 61.3 & 59.8 & 63.1 & 60.5 & $61.1 \mathrm{c}$ \\
\hline Achook & 45.1 & 49.4 & 55.9 & 57.4 & 58.9 & 54.0 & $53.5 \mathrm{~d}$ \\
\hline Applaud & 39.6 & 41.6 & 41.4 & 43.7 & 43.8 & 44.3 & $42.4 \mathrm{e}$ \\
\hline Breef & 34.2 & 40.6 & 45.8 & 45.8 & 44.6 & 44.1 & $42.5 \mathrm{e}$ \\
\hline Admire + Kemesol & 73.7 & 82.2 & 84.6 & 87.7 & 86.2 & 84.4 & $83.1 \mathrm{~b}$ \\
\hline Admire + Achook & 80.0 & 84.8 & 85.8 & 89.9 & 90.3 & 89.3 & $86.7 \mathrm{ab}$ \\
\hline Admire + Applaud & 54.7 & 61.1 & 62.8 & 64.5 & 66.8 & 64.0 & $62.3 \mathrm{c}$ \\
\hline Admire + Breef & 51.1 & 55.7 & 54.9 & 57.0 & 59.2 & 57.2 & $55.9 \mathrm{~d}$ \\
\hline
\end{tabular}

Means followed by the same letters are not significantly different according to the $\operatorname{LSD}_{0.05}$.

Table5.A.Efficacy of certain treatments against lady beetle on the eggplant (season 2012)

\begin{tabular}{lccccccc}
\hline Treatments & \multicolumn{6}{c}{ \% Reduction } & Mean \\
\cline { 2 - 7 } & 1-day & 3-days & 5-days & 7-days & 9-days & 12-days & \\
\hline Admire & 56.4 & 61.3 & 62.8 & 61.7 & 58.1 & 58.3 & $59.8 \mathrm{a}$ \\
Kemesol & 33.2 & 35.6 & 37.1 & 36.8 & 38.9 & 40.8 & $37.1 \mathrm{~b}$ \\
Achook & 21.9 & 27.8 & 34.1 & 32.7 & 31.9 & 31.6 & $30.0 \mathrm{c}$ \\
Applaud & 26.7 & 30.8 & 35.8 & 39.2 & 40.6 & 39.5 & $35.4 \mathrm{~b}$ \\
Breef & 8.2 & 10.3 & 11.7 & 10.1 & 9.6 & 7.2 & $9.5 \mathrm{e}$ \\
Admire + Kemesol & 28.0 & 31.6 & 29.8 & 29.5 & 27.2 & 25.9 & $28.7 \mathrm{c}$ \\
Admire + Achook & 18.4 & 17.7 & 20.1 & 18.2 & 18.2 & 16.0 & $18.1 \mathrm{~d}$ \\
Admire + Applaud & 20.3 & 21.5 & 24.7 & 22.1 & 20.3 & 19.9 & $21.5 \mathrm{~d}$ \\
Admire + Breef & 15.6 & 20.1 & 21.4 & 19.5 & 18.3 & 17.8 & $18.8 \mathrm{~d}$ \\
\hline
\end{tabular}

Means followed by the same letters are not significantly different according to the $\mathrm{LSD}_{0.05}$. 
Table 5.B. Efficacy of certain treatments against lady beetle on the eggplant (season 2013)

\begin{tabular}{lccccccc}
\hline Treatments & \multicolumn{6}{c}{ \% Reduction } & Mean \\
\cline { 2 - 7 } & 1-day & 3-days & 5-days & 7-days & 9-days & 12-days & \\
\hline Admire & 54.0 & 58.7 & 60.2 & 61.3 & 56.9 & 55.1 & $57.7 \mathrm{a}$ \\
Kemesol & 30.8 & 31.3 & 35.8 & 35.7 & 34.2 & 35.9 & $34.0 \mathrm{~b}$ \\
Achook & 23.6 & 25.1 & 28.7 & 30.5 & 31.0 & 31.4 & $28.4 \mathrm{c}$ \\
Applaud & 23.1 & 27.3 & 32.0 & 33.7 & 34.5 & 34.3 & $30.8 \mathrm{bc}$ \\
Breef & 10.0 & 11.8 & 9.2 & 8.5 & 8.7 & 5.4 & $8.9 \mathrm{e}$ \\
Admire + Kemesol & 25.2 & 28.8 & 27.1 & 27.8 & 25.9 & 23.6 & $26.4 \mathrm{c}$ \\
Admire + Achook & 16.6 & 16.1 & 18.8 & 18.5 & 16.4 & 15.9 & $17.1 \mathrm{~d}$ \\
Admire + Applaud & 18.0 & 19.4 & 21.3 & 22.6 & 18.8 & 17.2 & $19.6 \mathrm{~d}$ \\
Admire + Breef & 12.8 & 15.0 & 17.7 & 16.1 & 15.6 & 15.2 & $15.4 \mathrm{~d}$ \\
\hline
\end{tabular}

Means followed by the same letters are not significantly different according to the $\mathrm{LSD}_{0.05}$.

Table 6.A. Efficacy of certain treatments against Aphid lion on the eggplant (season 2012)

\begin{tabular}{|c|c|c|c|c|c|c|c|}
\hline \multirow[t]{2}{*}{ Treatments } & \multicolumn{6}{|c|}{ \% Reduction } & \multirow[t]{2}{*}{ Mean } \\
\hline & 1-day & 3-days & 5-days & 7-days & 9-days & 12-days & \\
\hline Admire & 24.1 & 26.6 & 27.7 & 26.3 & 24.2 & 23.9 & $25.5 \mathrm{a}$ \\
\hline Kemesol & 13.5 & 15.0 & 15.4 & 17.1 & 16.6 & 15.3 & $15.5 \mathrm{~b}$ \\
\hline Achook & 9.1 & 10.4 & 10.7 & 12.9 & 11.6 & 9.2 & $10.7 \mathrm{c}$ \\
\hline Applaud & 14.1 & 16.5 & 17.3 & 16.0 & 15.2 & 13.9 & $15.5 \mathrm{~b}$ \\
\hline Breef & 2.7 & 5.6 & 5.8 & 4.5 & 6.4 & 4.9 & $5.0 \mathrm{~d}$ \\
\hline Admire + Kemesol & 10.3 & 12.6 & 13.5 & 11.9 & 11.8 & 9.3 & $11.6 \mathrm{bc}$ \\
\hline Admire + Achook & 11.3 & 13.8 & 12.5 & 11.7 & 10.4 & 9.4 & $11.5 \mathrm{bc}$ \\
\hline Admire + Applaud & 12.0 & 13.4 & 13.5 & 11.7 & 10.6 & 9.1 & $11.7 \mathrm{bc}$ \\
\hline Admire + Breef & 5.2 & 5.6 & 6.9 & 5.1 & 4.5 & 4.0 & $5.2 \mathrm{~d}$ \\
\hline
\end{tabular}

Means followed by the same letters are not significantly different according to the $\mathrm{LSD}_{0.05}$.

Table 6.B. Efficacy of certain treatments against Aphid lion on the eggplant (season 2013)

\begin{tabular}{|c|c|c|c|c|c|c|c|}
\hline \multirow[t]{2}{*}{ Treatments } & \multicolumn{6}{|c|}{$\%$ Reduction } & \multirow[t]{2}{*}{ Mean } \\
\hline & 1-day & 3-days & 5-days & 7-days & 9-days & 12-days & \\
\hline Admire & 20.5 & 22.8 & 22.0 & 24.3 & 23.7 & 22.2 & $22.6 \mathrm{a}$ \\
\hline Kemesol & 10.1 & 12.4 & 11.6 & 13.6 & 13.2 & 12.3 & $12.2 \mathrm{~b}$ \\
\hline Achook & 6.8 & 7.6 & 7.9 & 8.2 & 8.3 & 6.5 & $7.6 \mathrm{bc}$ \\
\hline Applaud & 12.5 & 13.2 & 13.8 & 14.7 & 13.6 & 13.2 & $13.5 \mathrm{~b}$ \\
\hline Breef & 2.1 & 2.7 & 2.1 & 3.2 & 3.5 & 2.7 & $2.7 \mathrm{~d}$ \\
\hline Admire + Kemesol & 7.9 & 8.3 & 8.6 & 8.2 & 7.1 & 7.0 & $7.9 \mathrm{bc}$ \\
\hline Admire + Achook & 8.2 & 8.6 & 9.0 & 8.5 & 7.0 & 7.5 & $8.1 \mathrm{bc}$ \\
\hline Admire + Applaud & 9.6 & 10.4 & 11.7 & 8.3 & 7.7 & 6.8 & $9.1 \mathrm{bc}$ \\
\hline Admire + Breef & 3.5 & 3.8 & 4.6 & 3.6 & 3.8 & 3.5 & $3.8 \mathrm{c}$ \\
\hline
\end{tabular}

Means followed by the same letters are not significantly different according to the $\mathrm{LSD}_{0.05}$.

\section{REFERENCES}

Abdel-Rahman, S. M. and H. K. Abou-Taleb. 2008. Field evaluation of certain control agents against some cotton sucking insects. Adv. Agric. Res., 13: 663-673.

Aslam, M.; M. Razaq; S. A. Shah and F. Ahmad. 2004. Comparative efficacy of different insecticides against sucking pests of cotton. J. Research Science, Bahauddin Zakariya Univ., 15: 53-58.

Brook, S. S. and P. C. Barnard. 1990. The Green Lacewing of World: A Generic Review (Neu. Chrysopidae). Bulletin of British Museum (Natural History), London, England.
Butler, G. D.; D. L. Coudriet and T. V. Hennebery. 1988. Toxicity and repellance of soybeans and cottonseed oils to the sweet potato whitefly and the aphids on cotton in greenhouse studies. Southwest Entomol., 13: 81-96.

Canard, M.; Y. Semeria and T. R. New. 1984. Biology of Chrysopidae. D. R. W. Junk Publishers.

CoStat Statistical Software, 1990. Microcomputer program analysis version 4.20, CoHort Software, Berkeley, CA.

El-Bessomy, M. A. E. 2003. Effect of certain insecticide, imidacloprid (Admire) against immature stage (nymph) of whitefly Bemisia tabaci (Genn.) infesting tomato plants. J. Pest Cont. Environ. Sci., 11: 45 - 52. 
Fraser, H. 2005. Reduced-risk pesticides and Biopesticides. In: OMAFRA. Government of Ontario.

Gordon, R. D. 1985. The Coccinellidae (Coleoptera) of America north of Mexico. J. New York Entomological Society, 93: 1-912.

Hameed, M.; M. A. Murtaza and M. A. Bhatti. 1997. Relative efficacy of new insecticides against insect pest complex of cotton. Pak. Entomol., 19: 70-72.

Henderson, C. F. and E. W. Telton. 1955. Tests with acaricides against the brown wheat mite. J. Econ. Entomol., 48: 157-161.

Horowitz, A. R.; Z. Mendelson; P. G. Weintraub and I. Ishaaya. 1998. Comparative toxicity of foliar and systemic applications of acetamiprid and imidacloprid against the cotton whitefly, Bemisia tabaci (Hemiptera: Aleyrodidae). Bulletin of Entomological Research, 88: 437 - 442.

Kalloo, G. 1993. Eggplant (Solanum melongena). In: Kalloo, G. (Ed.), Genetic Improvement of Vegetable Crops. Pergamon Press, Oxford, pp. 587-604.

Kashyap, V.; S. V. Kumar; C. Collonnier; F. Fusari; R. Haicour; G. L. Rotino; D. Sihachakr and M. V. Rajam. 2003. Biotechnology of eggplant. Scientia Horticulturae, 97: 1-25.
Khan, R. 1979. Solanum melanogena and its ancestral forms. In: Hawkes, J., Lester, R. N., Skelding, A. D. (Eds.), The Biology and Taxonomy of Solanaceae. Academic Press, London, pp. 629-636.

Motohiro, T. and J. E. Casida. 2003. Selective toxicity of new nicotinoids attributable to specificity of insect and mammalian nicotinic receptors. Annu. Rev. Entomol., 48: $339-364$.

Pedigo, L. P. and M. E. Rice. 2006. Entomology and Pest Management. Pearson Prentice Hall.

Ruberson, J. R.; H. Nemoto and Y. Hirose. 1998. Pesticides and conservation of natural enemies in pest management. In: Barbosa, P. (ed), Conservation Biological Control. Academic Press, San Diego, CA, pp. 207-220.

Shehata, S. A.; M. A. Abd El-Latief and H. K. Abou-Taleb. 2009. Evaluation of some natural insecticides against some insects infesting cucumber and their predatory insect (Syrphus corollae F.) in the field. Alex. J. Agric. Res., 54: 49-55.

Wahla, M. A.; M. Tufail and P. Iqbal. 1997. The comparative effectiveness of different doses of Confidor 200SL and Tamaron 600SL against cotton thrips, Thrips tabaci Lind. on FH-582, cotton. Pak. Entomol., 19: 8-10.

Yadav, S. R. and K. C. Kumawat. 2013. Assessment of crop losses due to insect pests of brinjal, Solanum melongena L. Insect Environment, 19: 16-18. 


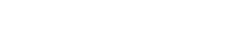

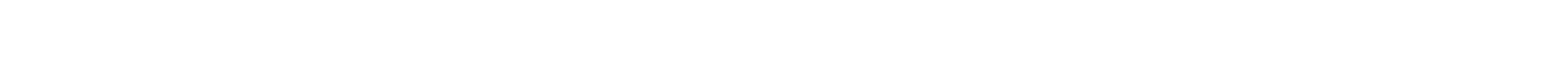
درلسة التأثير الجلنب لهنه الهولل على الأعداء اللبيعيه

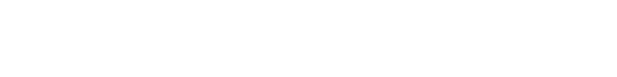

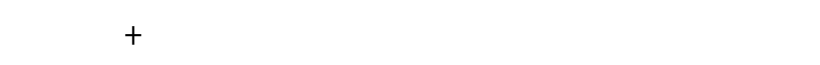

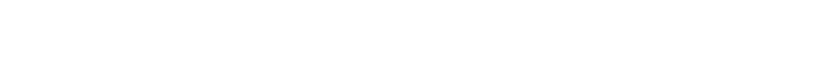

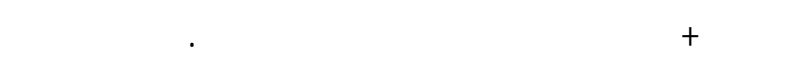

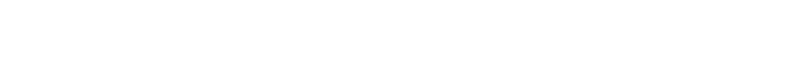

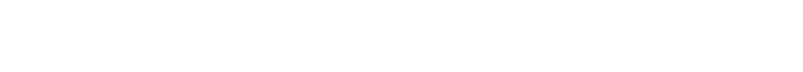

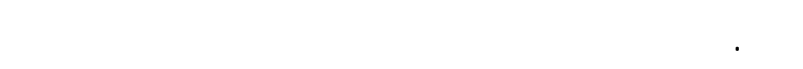

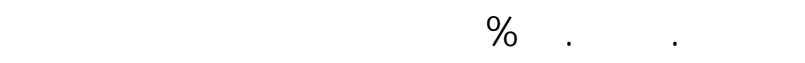

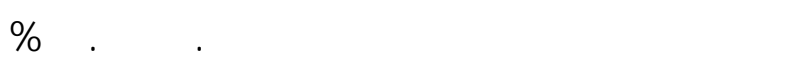

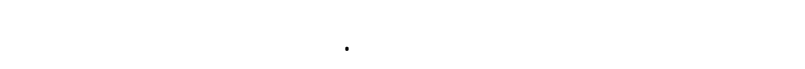

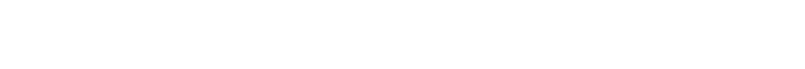

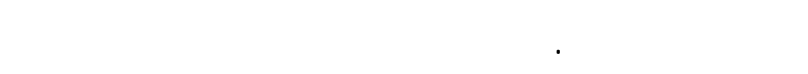

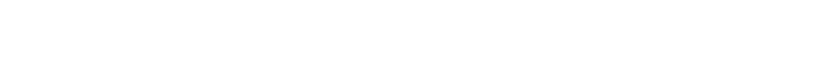

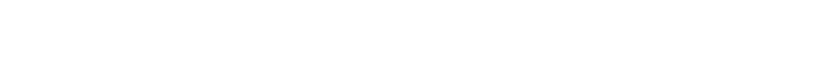

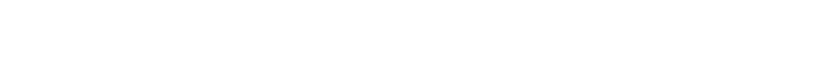
تأثيرات جانبيه ضعيفه على المشرات النافعه.

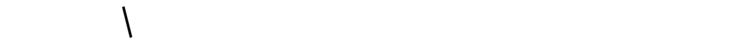

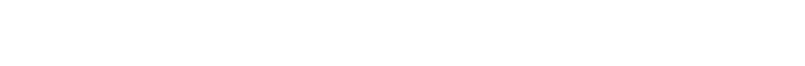

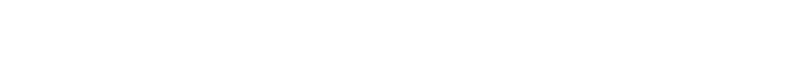
على البانجان. أيضا قم ققيم التأثير الجانبى لهذه المعلملات

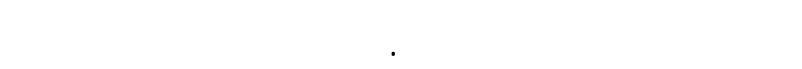

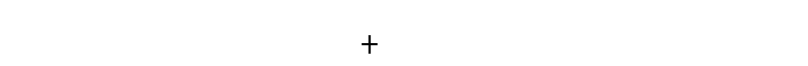

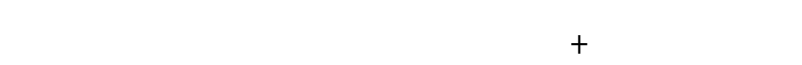

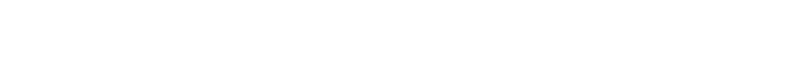

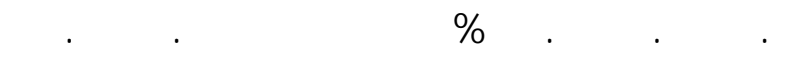

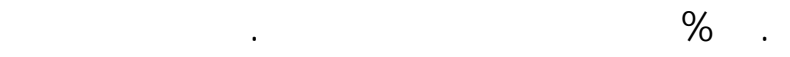

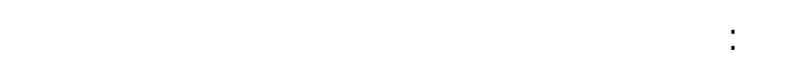

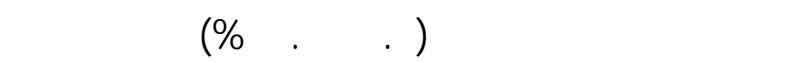

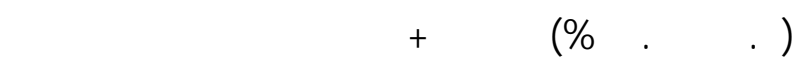

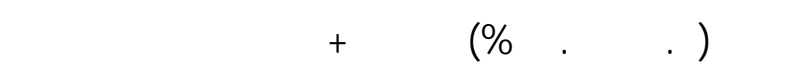

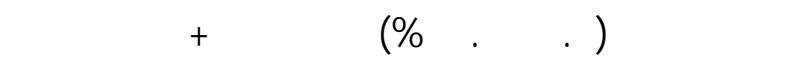

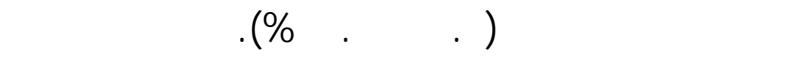

